\title{
STATISTICS OF $K$-GROUPS MODULO $p$ FOR THE RING OF INTEGERS OF A VARYING QUADRATIC NUMBER FIELD
}

\author{
BRUCE W. JORDAN, ZEV KLAGSBRUN, BJORN POONEN, CHRISTOPHER SKINNER, \\ AND YEVGENY ZAYTMAN
}

\begin{abstract}
For each odd prime $p$, we conjecture the distribution of the $p$-torsion subgroup of $K_{2 n}\left(\mathcal{O}_{F}\right)$ as $F$ ranges over real quadratic fields, or over imaginary quadratic fields. We then prove that the average size of the 3-torsion subgroup of $K_{2 n}\left(\mathcal{O}_{F}\right)$ is as predicted by this conjecture.
\end{abstract}

\section{INTRODUCTION}

The original Cohen-Lenstra heuristics CL84 predicted, for each prime $p \neq 2$, the distribution of the $p$-primary part of $\mathrm{Cl}(F)$ as $F$ varied over quadratic fields of a given signature. More recent work developed heuristics for other families of groups, including class groups of higher degree number fields [CM90, Picard groups of function fields [FW89, TateShafarevich groups of elliptic curves [Del01, Del07, DJ14, Selmer groups of elliptic curves [PR12,BKLPR15, and Galois groups of nonabelian unramified extensions of number fields and function fields [BBH17, BW17].

Let $\mathcal{F}$ be a number field. Let $\mathcal{O}_{\mathcal{F}}$ be the ring of integers of $\mathcal{F}$. For $m \geq 0$, the $K$-group $K_{m}\left(\mathcal{O}_{\mathcal{F}}\right)$ is a finitely generated abelian group. It is finite when $m$ is even and positive: see Wei05, Theorem 7]. Our goal is to study, for a fixed $m$ and odd prime $p$, the $p$-torsion subgroup $K_{m}\left(\mathcal{O}_{\mathcal{F}}\right)_{p}$ as $\mathcal{F}$ varies in a family of number fields, always ordered by absolute value of the discriminant. As described in Section $6, K_{m}\left(\mathcal{O}_{\mathcal{F}}\right)_{p}$ is well understood for odd $m$. Therefore we focus on the case $m=2 n$. Now suppose that $\mathcal{F}$ is a quadratic field $F$. The action of $\operatorname{Gal}(F / \mathbb{Q})$ decomposes $K_{2 n}\left(\mathcal{O}_{F}\right)_{p}$ into + and - parts, and we will see that the + part is $K_{2 n}(\mathbb{Z})_{p}$, independent of $F$. Therefore we focus on the variation of the - part.

A Cohen-Lenstra style heuristic will lead us to the following conjecture, involving the constants

$$
\alpha_{p, u, r}:=\frac{\prod_{i=r+1}^{\infty}\left(1-p^{-i}\right)}{p^{r(u+r)} \prod_{i=1}^{r+u}\left(1-p^{-i}\right)}
$$

for nonnegative integers $u$ and $r$ :

Conjecture 1.1. Fix $n \geq 1$ and an odd prime $p$ and $r \geq 0$. As $F$ ranges over all real (resp. imaginary) quadratic fields, Prob $\left(\operatorname{dim}_{\mathbb{F}_{p}} K_{2 n}\left(\mathcal{O}_{F}\right)_{p}^{-}=r\right)$ is given in the following table by the entry in the row determined by $n$ and column determined by the signature:

Date: January 28, 2019.

2010 Mathematics Subject Classification. Primary 11R70; Secondary 11R29, 19D50, 19 F99.

Key words and phrases. algebraic K-theory, ring of integers, class group, Cohen-Lenstra heuristics.

B.P. was supported in part by National Science Foundation grant DMS-1601946 and Simons Foundation grants \#402472 (to Bjorn Poonen) and \#550033. C.S. was supported in part by National Science Foundation grant DMS-1301842 and by the Simons Investigator grant \#376203 from the Simons Foundation. 


\begin{tabular}{c|c||c|c} 
& & real & imaginary \\
\hline \hline$n$ even & $n \equiv 0(\bmod p-1)$ & $\frac{1}{2}\left(\frac{p}{p+1} \alpha_{p, 2, r-1}+\frac{p+2}{p+1} \alpha_{p, 1, r}\right)$ & $\frac{1}{2}\left(\frac{p}{p+1} \alpha_{p, 1, r-1}+\frac{p+2}{p+1} \alpha_{p, 0, r}\right)$ \\
& $n \equiv \frac{p-1}{2}(\bmod p-1)$ & $\frac{1}{2}\left(\frac{1}{p+1} \alpha_{p, 2, r-1}+\frac{2 p+1}{p+1} \alpha_{p, 1, r}\right)$ & $\frac{1}{2}\left(\frac{1}{p+1} \alpha_{p, 1, r-1}+\frac{2 p+1}{p+1} \alpha_{p, 0, r}\right)$ \\
& all other cases & $\alpha_{p, 1, r}$ & $\alpha_{p, 0, r}$ \\
\hline$n$ odd & $n \equiv \frac{p-1}{2}(\bmod p-1)$ & $\frac{1}{2}\left(\frac{1}{p+1} \alpha_{p, 1, r-1}+\frac{2 p+1}{p+1} \alpha_{p, 0, r}\right)$ & $\frac{1}{2}\left(\frac{1}{p+1} \alpha_{p, 2, r-1}+\frac{2 p+1}{p+1} \alpha_{p, 1, r}\right)$ \\
& all other cases & $\alpha_{p, 0, r}$ & $\alpha_{p, 1, r}$.
\end{tabular}

To pass from the distribution of $\operatorname{dim}_{\mathbb{F}_{p}} K_{2 n}\left(\mathcal{O}_{F}\right)_{p}^{-}$to that of $\operatorname{dim}_{\mathbb{F}_{p}} K_{2 n}\left(\mathcal{O}_{F}\right)_{p}$ itself, add the constant $\operatorname{dim}_{\mathbb{F}_{p}} K_{2 n}(\mathbb{Z})_{p}$, which can be expressed in terms of a class group (see Section 5 ).

Conjecture 1.1 implies an average order for $K_{2 n}\left(\mathcal{O}_{F}\right)_{p}$ as $F$ varies over all real or imaginary fields (see Conjecture 8.8). We prove that this conjectured average order is correct for $p=3$ :

Theorem 1.2. Fix $n \geq 1$. The average order of $K_{2 n}\left(\mathcal{O}_{F}\right)_{3}$ as $F$ ranges over real (resp. imaginary) quadratic fields is as follows:

\begin{tabular}{c||c|c} 
& real & imaginary \\
\hline \hline$n$ even & $25 / 12$ & $11 / 4$ \\
\hline$n$ odd & $9 / 4$ & $19 / 12$.
\end{tabular}

Remark 1.3. By Theorem 5.1, $K_{2 n}(\mathbb{Z})_{3}=0$ for all $n$ since $\mathbb{Q}\left(\zeta_{3}\right)$ has class number 1 . Thus $K_{2 n}\left(\mathcal{O}_{F}\right)_{3}^{-}=K_{2 n}\left(\mathcal{O}_{F}\right)_{3}$ for all $n$.

Remark 1.4. Theorem 1.2 is an analogue of the Davenport-Heilbronn theorem giving the average order of $\mathrm{Cl}(F)_{3}$ as $F$ varies over all real or imaginary quadratic fields [DH71, Theorem 3].

Remark 1.5. After this article was written, the second author proved an analogue of Theorem 1.2 for $K_{2 n}\left(\mathcal{O}_{\mathcal{F}}\right)_{2}$ as $\mathcal{F}$ varies over cubic fields [Kla17b].

1.1. Methods. The $p$-torsion subgroup $G_{p}$ of a finite abelian group $G$ has the same $\mathbb{F}_{p^{-}}$ dimension as $G / p:=G / p G$; therefore we study $K_{2 n}\left(\mathcal{O}_{F}\right) / p$. The latter is isomorphic to an étale cohomology group $H_{\text {ét }}^{2}\left(\mathcal{O}_{F}[1 / p], \mu_{p}^{\otimes(n+1)}\right)$, which we relate to isotypic components of the class group and Brauer group of $\mathcal{O}_{E}[1 / p]$, where $E:=F\left(\zeta_{p}\right)$. The Brauer group can be computed explicitly, and we develop heuristics for the class groups; combining these gives the conjectural distribution of $K_{2 n}\left(\mathcal{O}_{F}\right)_{p}$.

In the case $p=3$, the isotypic components of $\mathrm{Cl}\left(\mathcal{O}_{E}[1 / p]\right)$ are related to $\mathrm{Cl}\left(\mathcal{O}_{K}[1 / p]\right)$ for quadratic fields $K$. The average order of the latter class groups can be computed unconditionally by using a strategy of Davenport and Heilbronn, which we refine using recent work of Bhargava, Shankar, and Tsimerman, to control averages in subfamilies with prescribed local behavior at 3 . This yields unconditional results on the average order of $K_{2 n}\left(\mathcal{O}_{F}\right)_{3}$.

1.2. Prior work. As far as we know, Cohen-Lenstra style conjectures have not been proposed for $K$-groups before, but some results on the distribution of $K_{2}\left(\mathcal{O}_{F}\right)$ have been proved.

Guo Guo09 proved that 4-ranks of $K_{2}\left(\mathcal{O}_{F}\right)$ for quadratic fields $F$ follow a Cohen-Lenstra distribution, just as Fouvry and Klüners proved for 4-ranks of $\mathrm{Cl}\left(\mathcal{O}_{F}\right)$ [FK07]. Studying 4ranks is natural, since the 2-rank of $K_{2}\left(\mathcal{O}_{F}\right)$ for a quadratic field $F$ is determined by genus theory just as the 2-rank of $\mathrm{Cl}\left(\mathcal{O}_{F}\right)$ is (see [BS82, for example). 
Similar results on the 3-ranks of $K_{2}\left(\mathcal{O}_{L}\right)$ for cyclic cubic fields $L$ are due to Cheng, Guo, and Qin [CGQ14. In addition, Browkin showed that Cohen-Martinet heuristics suggest a conjecture on $\operatorname{Prob}\left(3 \mid \# K_{2}\left(\mathcal{O}_{F}\right)\right)$ as $F$ ranges over quadratic fields of fixed signature [Bro00].

1.3. Notation. If $G$ is an abelian group and $n \geq 1$, let $G_{n}:=\{g \in G: n g=0\}$ and $G / n:=G / n G$. For any $k$-representation $V$ of a finite group $G$ such that char $k \nmid \# G$, and for any irreducible $k$-representation $\chi$ of $G$, let $V^{\chi}$ be the $\chi$-isotypic component.

Throughout the paper, $p$ is an odd prime, $\mathcal{F}$ is an arbitrary number field, $\overline{\mathcal{F}}$ is an algebraic closure of $\mathcal{F}$, the element $\zeta \in \overline{\mathcal{F}}$ is a primitive $p$ th root of unity, and $\mathcal{E}:=\mathcal{F}\left(\zeta_{p}\right)$. Later we specialize $\mathcal{F}$ to a quadratic field $F$ and define $E:=F\left(\zeta_{p}\right)$.

Let $\mathcal{O}_{\mathcal{F}}$ be the ring of integers of $\mathcal{F}$. If $S$ is a finite set of places of $\mathcal{F}$ containing all the archimedean places, define the ring of $S$-integers $\mathcal{O}_{S}:=\{x \in \mathcal{F}: v(x) \geq 0$ for all $v \notin S\}$. Let $d_{\mathcal{F}} \in \mathbb{Z}$ be the discriminant of $\mathcal{F}$. Let $\mu(\mathcal{F})$ be the group of roots of unity in $\mathcal{F}$.

If $\mathcal{O}$ is a Dedekind ring, let $\mathrm{Cl}(\mathcal{O})$ denote its class group, and let $\operatorname{Br}(\mathcal{O})$ be its (cohomological) Brauer group, defined as $H_{\text {ét }}^{2}\left(\operatorname{Spec} \mathcal{O}, \mathbb{G}_{m}\right)$ [Poo17, Definition 6.6.4]. From now on, all cohomology is étale cohomology, and we drop the subscript ét.

\section{From $K$-Theory to Class groups and Brauer groups}

In this section, following Tate's argument for $K_{2}$ [Tat76], we relate the even $K$-groups to more concrete groups: class groups and Brauer groups.

Theorem 2.1 (Corollary 71 in [Wei05]). For any number field $\mathcal{F}$ and any $n \geq 1$,

$$
K_{2 n}\left(\mathcal{O}_{\mathcal{F}}\right) / p \simeq H^{2}\left(\mathcal{O}_{\mathcal{F}}[1 / p], \mu_{p}^{\otimes(n+1)}\right) .
$$

Lemma 2.2. There is a canonical exact sequence

$$
0 \longrightarrow \mathrm{Cl}\left(\mathcal{O}_{\mathcal{F}}[1 / p]\right) / p \longrightarrow H^{2}\left(\mathcal{O}_{\mathcal{F}}[1 / p], \mu_{p}\right) \longrightarrow \operatorname{Br}\left(\mathcal{O}_{\mathcal{F}}[1 / p]\right)_{p} \longrightarrow 0 .
$$

Proof. Consider the exact sequence

$$
1 \rightarrow \mu_{p} \rightarrow \mathbb{G}_{m} \stackrel{p}{\rightarrow} \mathbb{G}_{m} \rightarrow 1
$$

of sheaves on $\left(\operatorname{Spec} \mathcal{O}_{\mathcal{F}}[1 / p]\right)_{\text {ét }}$. Take the associated long exact sequence of cohomology, and substitute $H^{1}\left(\mathcal{O}_{\mathcal{F}}[1 / p], \mathbb{G}_{m}\right)=\operatorname{Pic}\left(\mathcal{O}_{\mathcal{F}}[1 / p]\right)=\mathrm{Cl}\left(\mathcal{O}_{\mathcal{F}}[1 / p]\right)$ and $H^{2}\left(\mathcal{O}_{\mathcal{F}}[1 / p], \mathbb{G}_{m}\right)=$ $\operatorname{Br}\left(\mathcal{O}_{\mathcal{F}}[1 / p]\right)$.

Lemma 2.3. Let $\mathcal{F}^{\prime} / \mathcal{F}$ be a finite Galois extension of degree prime to $p$. Let $i \geq 0$ and $r \in \mathbb{Z}$. Then

$$
H^{i}\left(\mathcal{O}_{\mathcal{F}}[1 / p], \mu_{p}^{\otimes r}\right)=H^{i}\left(\mathcal{O}_{\mathcal{F}^{\prime}}[1 / p], \mu_{p}^{\otimes r}\right)^{\operatorname{Gal}\left(\mathcal{F}^{\prime} / \mathcal{F}\right)} .
$$

Proof. In the Hochschild-Serre spectral sequence

$$
H^{i}\left(\operatorname{Gal}\left(\mathcal{F}^{\prime} / \mathcal{F}\right), H^{j}\left(\mathcal{O}_{\mathcal{F}^{\prime}}[1 / p], \mu_{p}^{\otimes r}\right)\right) \Longrightarrow H^{i+j}\left(\mathcal{O}_{\mathcal{F}}[1 / p], \mu_{p}^{\otimes r}\right),
$$

the groups $H^{i}\left(\operatorname{Gal}\left(\mathcal{F}^{\prime} / \mathcal{F}\right), H^{j}\left(\mathcal{O}_{\mathcal{F}^{\prime}}[1 / p], \mu_{p}^{\otimes r}\right)\right)$ for $i>0$ are 0 because they are killed by both $\# \operatorname{Gal}\left(\mathcal{F}^{\prime} / \mathcal{F}\right)$ and $p$.

We now specialize $\mathcal{F}^{\prime}$ to $\mathcal{E}:=\mathcal{F}\left(\zeta_{p}\right)$. The action of $\operatorname{Gal}(\mathcal{E} / \mathcal{F})$ on the $p$ th roots of 1 defines an injective homomorphism $\chi_{1}: \operatorname{Gal}(\mathcal{E} / \mathcal{F}) \rightarrow(\mathbb{Z} / p \mathbb{Z})^{\times}$. For $m \in \mathbb{Z}$, composing $\chi_{1}$ with the $m$ th power map on $(\mathbb{Z} / p \mathbb{Z})^{\times}$yields another 1 -dimensional $\mathbb{F}_{p}$-representation of $\operatorname{Gal}(\mathcal{E} / \mathcal{F})$; call it $\chi_{m}$. 
Lemma 2.4. There is a split exact sequence

$$
0 \rightarrow\left(\mathrm{Cl}\left(\mathcal{O}_{\mathcal{E}}[1 / p]\right) / p\right)^{\chi_{-n}} \rightarrow H^{2}\left(\mathcal{O}_{\mathcal{F}}[1 / p], \mu_{p}^{\otimes(n+1)}\right) \rightarrow\left(\operatorname{Br}\left(\mathcal{O}_{\mathcal{E}}[1 / p]\right)_{p}\right)^{\chi_{-n}} \rightarrow 0 .
$$

Proof. First, we have

$$
\begin{aligned}
H^{2}\left(\mathcal{O}_{\mathcal{F}}[1 / p], \mu_{p}^{\otimes(n+1)}\right) & =H^{2}\left(\mathcal{O}_{\mathcal{E}}[1 / p], \mu_{p}^{\otimes(n+1)}\right)^{\operatorname{Gal}(\mathcal{E} / \mathcal{F})} \quad(\text { Lemma 2.3) } \\
& =\left(H^{2}\left(\mathcal{O}_{\mathcal{E}}[1 / p], \mu_{p}\right) \otimes \mu_{p}^{\otimes n}\right)^{\operatorname{Gal}(\mathcal{E} / \mathcal{F})} \quad\left(\text { since } \mu_{p} \subset \mathcal{E}\right) \\
& =H^{2}\left(\mathcal{O}_{\mathcal{E}}[1 / p], \mu_{p}\right)^{\chi-n} \quad\left(\text { since } \mu_{p}^{\otimes n} \simeq \chi_{n}\right) .
\end{aligned}
$$

On the other hand, Lemma 2.2 for $\mathcal{E}$ yields a sequence of $\operatorname{Gal}(\mathcal{E} / \mathcal{F})$-representations

$$
0 \longrightarrow \mathrm{Cl}\left(\mathcal{O}_{\mathcal{E}}[1 / p]\right) / p \longrightarrow H^{2}\left(\mathcal{O}_{\mathcal{E}}[1 / p], \mu_{p}\right) \longrightarrow \operatorname{Br}\left(\mathcal{O}_{\mathcal{E}}[1 / p]\right)_{p} \longrightarrow 0,
$$

which splits by Maschke's theorem. Take $\chi_{-n}$-isotypic components, and substitute (1) in the middle.

Substituting Theorem 2.1 into Lemma 2.4 yields the main result of this section:

Theorem 2.5. For each $n \geq 1$,

$$
K_{2 n}\left(\mathcal{O}_{\mathcal{F}}\right) / p \simeq\left(\mathrm{Cl}\left(\mathcal{O}_{\mathcal{E}}[1 / p]\right) / p\right)^{\chi-n} \oplus\left(\operatorname{Br}\left(\mathcal{O}_{\mathcal{E}}[1 / p]\right)_{p}\right)^{\chi-n} .
$$

\section{Even $K$-groups of THE RING OF INTEGERS OF A QUADRATIC FIELD}

Let $p^{*}=(-1)^{(p-1) / 2} p$, so $\mathbb{Q}\left(\sqrt{p^{*}}\right)$ is the degree 2 subfield of $\mathbb{Q}\left(\zeta_{p}\right)$. From now on, $F$ is a degree 2 extension of $\mathbb{Q}$ not equal to $\mathbb{Q}\left(\sqrt{p^{*}}\right)$. Thus $F=\mathbb{Q}(\sqrt{d})$ for some $d \in \mathbb{Q}^{\times}$such that $d$ and $p^{*}$ are independent in $\mathbb{Q}^{\times} / \mathbb{Q}^{\times 2}$. Let $E=F\left(\zeta_{p}\right)$. Then

$$
\operatorname{Gal}(E / \mathbb{Q}) \simeq(\mathbb{Z} / p \mathbb{Z})^{\times} \times\{ \pm 1\} .
$$

Let $\tau$ be the generator of $\operatorname{Gal}\left(E / \mathbb{Q}\left(\zeta_{p}\right)\right)=\{ \pm 1\}$, so $\tau$ restricts to the generator of $\operatorname{Gal}(F / \mathbb{Q})$. The action of $\tau$ decomposes $K_{2 n}\left(\mathcal{O}_{F}\right) / p$ into + and - eigenspaces. Let $\chi_{-n,-1}: G \rightarrow \mathbb{F}_{p}^{\times}$be such that $\left.\chi_{-n,-1}\right|_{\operatorname{Gal}(E / F)}=\chi_{-n}$ and $\chi_{-n,-1}(\tau)=-1$.

Theorem 3.1. For each $n \geq 1$,

$$
\begin{aligned}
& \left(K_{2 n}\left(\mathcal{O}_{F}\right) / p\right)^{+} \simeq K_{2 n}(\mathbb{Z}) / p \\
& \left(K_{2 n}\left(\mathcal{O}_{F}\right) / p\right)^{-} \simeq\left(\operatorname{Cl}\left(\mathcal{O}_{E}[1 / p]\right) / p\right)^{\chi_{-n,-1}} \oplus\left(\operatorname{Br}\left(\mathcal{O}_{E}[1 / p]\right)_{p}\right)^{\chi_{-n,-1}} .
\end{aligned}
$$

Proof. To obtain the first statement, use Theorem 2.1 to rewrite each term as an étale cohomology group and apply Lemma 2.3 with $\mathcal{F}^{\prime} / \mathcal{F}$ there being $F / \mathbb{Q}$. To obtain the second, take minus parts in Theorem 2.5.

\section{Brauer groups}

The goal of this section is to determine the rightmost term in Theorem 3.1.

Lemma 4.1 (Equation (6.9.5) in [Poo17]). Let $\mathcal{F}$ and $\mathcal{O}_{S}$ be as in Section 1.3. Let $r_{1}$ be the number of real places of $\mathcal{F}$. Then there is an exact sequence

$$
0 \longrightarrow \operatorname{Br} \mathcal{O}_{S} \longrightarrow\left(\frac{1}{2} \mathbb{Z} / \mathbb{Z}\right)^{r_{1}} \oplus \bigoplus_{\text {finite } v \in S} \mathbb{Q} / \mathbb{Z} \stackrel{\text { sum }}{\longrightarrow} \mathbb{Q} / \mathbb{Z}
$$

Corollary 4.2. We have $\operatorname{Br}\left(\mathcal{O}_{S}\right)_{p} \simeq(\mathbb{Z} / p \mathbb{Z})_{\text {sum } 0}^{\text {\{finite } v \in S\}}$, where the "sum 0 " subscript denotes the subgroup of elements whose sum is 0 . 
Corollary 4.3. We have $\operatorname{Br}\left(\mathbb{Z}\left[\zeta_{p}, 1 / p\right]\right)_{p}=0$.

Proof. There is only one prime above $p$ in $\mathbb{Z}\left[\zeta_{p}\right]$.

Proposition 4.4. Let $F$ and $E$ be as in Section [3. Then

$$
\left(\operatorname{Br}\left(\mathcal{O}_{E}[1 / p]\right)_{p}\right)^{\chi-n,-1}= \begin{cases}\mathbb{Z} / p \mathbb{Z}, & \text { if } n \equiv 0(\bmod p-1) \text { and } d \in \mathbb{Q}_{p}^{\times 2} ; \\ \mathbb{Z} / p \mathbb{Z}, & \text { if } n \equiv \frac{p-1}{2}(\bmod p-1) \text { and } p^{*} d \in \mathbb{Q}_{p}^{\times 2} \\ 0, & \text { in all other cases. }\end{cases}
$$

Proof. The hypothesis implies that $F$ is not the quadratic subfield $\mathbb{Q}\left(\sqrt{p^{*}}\right)$ of $\mathbb{Q}\left(\zeta_{p}\right)$. Thus $E$ is the compositum of linearly disjoint extensions $\mathbb{Q}\left(\zeta_{p}\right)$ and $F$ over $\mathbb{Q}$, and $\operatorname{Gal}(E / \mathbb{Q}) \simeq$ $(\mathbb{Z} / p \mathbb{Z})^{\times} \times\{ \pm 1\}$. Let $\mathfrak{p}$ be a prime of $E$ lying above $p$. Let $D \leq \operatorname{Gal}(E / \mathbb{Q})$ be the decomposition group of $\mathfrak{p}$. Since $p$ totally ramifies in $\mathbb{Q}\left(\zeta_{p}\right) / \mathbb{Q}$, we have $p-1 \mid \# D$. Let $S_{p}$ be the set of primes of $E$ lying above $p$, so $S_{p} \simeq \operatorname{Gal}(E / \mathbb{Q}) / D$, which by the previous sentence is of size 1 or 2 ; it is 2 if and only if $p$ splits in one of the quadratic subfields of $E$. These quadratic subfields are $\mathbb{Q}\left(\sqrt{p^{*}}\right), F$, and the field $F^{\prime}=\mathbb{Q}\left(\sqrt{p^{*} d}\right)$, but $p$ is ramified in $\mathbb{Q}\left(\sqrt{p^{*}}\right)$. Thus by Corollary 4.2,

$$
\operatorname{Br}\left(\mathcal{O}_{E}[1 / p]\right)_{p}=(\mathbb{Z} / p \mathbb{Z})_{\text {sum } 0}^{S_{p}}= \begin{cases}\mathbb{Z} / p \mathbb{Z}, & \text { if } p \text { splits in } F \text { or } F^{\prime} \\ 0, & \text { otherwise }\end{cases}
$$

as an abelian group, and it remains to determine in the first case which character it is isomorphic to. We will compute the action of $\operatorname{Gal}(E / F) \simeq(\mathbb{Z} / p \mathbb{Z})^{\times}$and $\operatorname{Gal}\left(E / \mathbb{Q}\left(\zeta_{p}\right)\right) \simeq$ $\{ \pm 1\}$ separately.

If $p$ splits in $F$ (that is, $d \in \mathbb{Q}_{p}^{\times 2}$ ), then $D=\operatorname{Gal}(E / F)$, which acts trivially on $S_{p} \simeq$ $\operatorname{Gal}(E / \mathbb{Q}) / D$, so $\operatorname{Gal}(E / F)$ acts on $\operatorname{Br}\left(\mathcal{O}_{E}[1 / p]\right)_{p}$ as the trivial character $\chi_{0}$. If instead $p$ splits in $F^{\prime}$ (that is, $p^{*} d \in \mathbb{Q}_{p}^{\times 2}$ ), then $D \neq \operatorname{Gal}(E / F)$, so $\operatorname{Gal}(E / F)$ acts nontrivially on the two-element set $S_{p} \simeq \operatorname{Gal}(E / \mathbb{Q}) / D$, so $\operatorname{Gal}(E / F)$ acts on $\operatorname{Br}\left(\mathcal{O}_{E}[1 / p]\right)_{p}$ as the character $\operatorname{Gal}(E / F) \rightarrow\{ \pm 1\}$, which is $\chi_{(p-1) / 2}$.

Finally, consider the action of the generator $\tau$ of $\operatorname{Gal}\left(E / \mathbb{Q}\left(\zeta_{p}\right)\right) \simeq\{ \pm 1\}$ on $\operatorname{Br}\left(\mathcal{O}_{E}[1 / p]\right)_{p}$. Lemma 2.3 shows that the + eigenspace is $\operatorname{Br}\left(\mathbb{Z}\left[\zeta_{p}, 1 / p\right]\right)_{p}$, which is 0 by Corollary 4.3 . Thus $\operatorname{Br}\left(\mathcal{O}_{E}[1 / p]\right)_{p}$ equals its - eigenspace.

Remark 4.5. Let $d$ be a fundamental discriminant. Then $d \in \mathbb{Q}_{p}^{\times 2}$ if and only if $\left(\frac{d}{p}\right)=1$, and $p^{*} d \in \mathbb{Q}_{p}^{\times 2}$ if and only if $p \mid d$ and $\left(\frac{-d / p}{p}\right)=1$.

\section{Even $K$-groups of $\mathbb{Z}$}

Theorem 5.1. For each $n \geq 1$,

$$
K_{2 n}(\mathbb{Z}) / p \simeq\left(\mathrm{Cl}\left(\mathbb{Z}\left[\zeta_{p}\right]\right) / p\right)^{\chi_{-n}} .
$$

Proof. In Theorem 2.5 for $\mathcal{F}=\mathbb{Q}$, the Brauer term is 0 by Corollary 4.3, and $\mathrm{Cl}\left(\mathbb{Z}\left[\zeta_{p}, 1 / p\right]\right)=$ $\mathrm{Cl}\left(\mathbb{Z}\left[\zeta_{p}\right]\right)$ since the unique prime ideal above $p$ in $\mathbb{Z}\left[\zeta_{p}\right]$ is principal.

For $n \geq 1$, let

$$
\kappa_{2 n, p}:=\operatorname{dim}_{\mathbb{F}_{p}} K_{2 n}(\mathbb{Z}) / p=\operatorname{dim}_{\mathbb{F}_{p}}\left(\mathrm{Cl}\left(\mathbb{Z}\left[\zeta_{p}\right]\right) / p\right)^{\chi_{-n}}
$$


Remark 5.2. Assuming Vandiver's conjecture that $p \nmid \# \mathrm{Cl}\left(\mathbb{Z}\left[\zeta_{p}+\zeta_{p}^{-1}\right]\right)$ for every prime $p$, the $K$-groups of $\mathbb{Z}$ are known; see [Wei05, Section 5.9]. To state the results for even $K$-groups, let $B_{2 k} \in \mathbb{Q}$ be the $(2 k)$ th Bernoulli number, defined by

$$
\frac{t}{e^{t}-1}=1-\frac{t}{2}+\sum_{k=1}^{\infty} B_{2 k} \frac{t^{2 k}}{(2 k) !} .
$$

Let $c_{k}$ be the numerator of $\left|B_{2 k} /(4 k)\right|$. Then (see [Wei05, Corollary 107])

- Vandiver's conjecture implies that $K_{4 k}(\mathbb{Z})=0$ for all $k \geq 1$.

- For $k \geq 1$, the order of $K_{4 k-2}(\mathbb{Z})$ is $c_{k}$ if $k$ is even, and $2 c_{k}$ if $k$ is odd; moreover, Vandiver's conjecture implies that $K_{4 k-2}(\mathbb{Z})$ is cyclic.

In fact, for each prime $p$, Vandiver's conjecture for $p$ implies the conclusions above for the $p$-primary part of the $K$-groups. Thus Vandiver's conjecture for an odd prime $p$ implies that for any $n \geq 1$,

$$
\kappa_{2 n, p}= \begin{cases}1 & \text { if } n=2 k-1 \text { and } p \mid c_{k} ; \\ 0 & \text { otherwise }\end{cases}
$$

Moreover, Vandiver's conjecture is known for $p<163577856$ [BH11].

The smallest odd prime $p$ for which there exists $n$ such that $p \mid \# K_{2 n}(\mathbb{Z})$ is the smallest irregular prime, 37 , which divides $\# K_{2 n}(\mathbb{Z})$ if and only if $n \equiv 31(\bmod 36)$; thus $\kappa_{2 n, 37}$ is 1 if $n \equiv 31(\bmod 36)$, and 0 otherwise. Assuming Vandiver's conjecture, the smallest $n$ such that $\# K_{2 n}(\mathbb{Z})$ is divisible by an odd prime is $n=11$ : we have $K_{22}(\mathbb{Z}) \simeq \mathbb{Z} / 691 \mathbb{Z}$. See [Wei05, Example 96] for these and other examples.

\section{OdD $K$-GROUPS}

Proposition 6.1. For any number field $\mathcal{F}$, positive integer $i$, and odd prime $p$, we have

$$
K_{2 i-1}\left(\mathcal{O}_{\mathcal{F}}\right)_{p}= \begin{cases}\mathbb{Z} / p \mathbb{Z}, & \text { if }\left[\mathcal{F}\left(\zeta_{p}\right): \mathcal{F}\right] \text { divides } i ; \\ 0, & \text { otherwise. }\end{cases}
$$

Proof. Define the group

$$
\mu^{(i)}(\mathcal{F}):=\left\{\zeta \in \mu(\overline{\mathcal{F}}): \sigma^{i} \zeta=\zeta \text { for all } \sigma \in \operatorname{Gal}(\overline{\mathcal{F}} / \mathcal{F})\right\} .
$$

For $n \geq 1$, let $\zeta_{n} \in \overline{\mathcal{F}}$ be a primitive $n$th root of 1 , and let $H_{n}$ be the image of the restriction homomorphism $\operatorname{Gal}\left(\mathcal{F}\left(\zeta_{n}\right) / \mathcal{F}\right) \hookrightarrow \operatorname{Gal}\left(\mathbb{Q}\left(\zeta_{n}\right) / \mathbb{Q}\right) \simeq(\mathbb{Z} / n \mathbb{Z})^{\times}$, so $\# H_{n}=\left[\mathcal{F}\left(\zeta_{n}\right): \mathcal{F}\right]$. Then the following are equivalent:

- $\zeta_{n} \in \mu^{(i)}(\mathcal{F})$;

- $\sigma^{i} \zeta_{n} \equiv \zeta_{n}$ for all $\sigma \in \operatorname{Gal}(\overline{\mathcal{F}} / \mathcal{F})$;

- $a^{i}=1$ for all $a \in H_{n}$.

Now suppose that $n$ is a prime power $\ell^{e}$ for some prime $\ell$. Then $H_{n}$ contains a cyclic subgroup of index at most 2 (we allow the case $\ell=2$ ). The last condition above implies $\# H_{n} \mid 2 i$, which after multiplication by $[\mathcal{F}: \mathbb{Q}]$ becomes the statement that $\left[\mathcal{F}\left(\zeta_{n}\right): \mathbb{Q}\right]$ divides $2 i[\mathcal{F}: \mathbb{Q}]$, which implies that the integer $\phi(n):=\left[\mathbb{Q}\left(\zeta_{n}\right): \mathbb{Q}\right]$ divides $2 i[\mathcal{F}: \mathbb{Q}]$, which bounds $\phi(n)$ and hence $n$. Thus $\mu^{(i)}(\mathcal{F})$ contains $\ell^{e}$ th roots of 1 for only finitely many prime powers $\ell^{e}$, so it is finite. Define $w^{(i)}(\mathcal{F}):=\# \mu^{(i)}(\mathcal{F})$. 
By Theorem 70 in [Wei05, if $p$ is an odd prime, $K_{2 i-1}\left(\mathcal{O}_{\mathcal{F}}\right)_{p}$ is $\mathbb{Z} / p \mathbb{Z}$ or 0 , according to whether $p$ divides $w^{(i)}(\mathcal{F})$ or not. The previous paragraph shows that the latter condition is equivalent to $H_{p}$ being killed by $i$, and to $\# H_{p} \mid i$ since $H_{p}$ is cyclic (a subgroup of the cyclic $\left.\operatorname{group}(\mathbb{Z} / p \mathbb{Z})^{\times}\right)$. Finally, $\# H_{p}=\left[\mathcal{F}\left(\zeta_{p}\right): \mathcal{F}\right]$.

\section{Heuristics FOR CLASS GROUPS}

Let $A$ be an abelian extension of $\mathbb{Q}$. Suppose that the Galois group $G:=\operatorname{Gal}(A / \mathbb{Q})$ is of exponent dividing $p-1$. Let $I_{A}^{p}$ be the group of fractional ideals of $\mathcal{O}_{A}[1 / p]$, that is, the free abelian group on the set of finite primes of $A$ not lying above $p$. We have the standard exact sequence of $\mathbb{Z} G$-modules

$$
1 \rightarrow \mathcal{O}_{A}[1 / p]^{\times} \rightarrow A^{\times} \rightarrow I_{A}^{p} \rightarrow \mathrm{Cl}\left(\mathcal{O}_{A}[1 / p]\right) \rightarrow 0
$$

Let $S$ be a finite set of places of $\mathbb{Q}$ including $p$ and $\infty$. Let $S_{A}$ be the set of places of $A$ above $S$. We approximate (21) by using only $S_{A}$-units and ideals supported on $S_{A}$. Let $S_{A}^{p}$ be the set of places of $A$ above $S-\{p\}$. Let $\mathcal{O}_{A, S}$ be the ring of $S_{A}$-integers in $A$. Let $I_{A, S}^{p}$ be the free abelian group on the nonarchimedean places in $S_{A}^{p}$. If $S$ is large enough that the finite primes in $S_{A}^{p}$ generate $\operatorname{Cl}\left(\mathcal{O}_{A}[1 / p]\right)$, then we have an exact sequence of $\mathbb{Z} G$-modules

$$
1 \rightarrow \mathcal{O}_{A}[1 / p]^{\times} \rightarrow \mathcal{O}_{A, S}^{\times} \rightarrow I_{A, S}^{p} \rightarrow \mathrm{Cl}\left(\mathcal{O}_{A}[1 / p]\right) \rightarrow 0 .
$$

Dropping the first term and tensoring with $\mathbb{F}_{p}$ yields an exact sequence of $\mathbb{F}_{p} G$-modules

$$
\mathcal{O}_{A, S}^{\times} / p \rightarrow I_{A, S}^{p} / p \rightarrow \mathrm{Cl}\left(\mathcal{O}_{A}[1 / p]\right) / p \rightarrow 0 .
$$

Let $\chi$ be an irreducible $\mathbb{F}_{p}$-representation of $G$; our assumption on $G$ guarantees that $\chi$ is 1-dimensional. Taking $\chi$-isotypic components yields

$$
\left(\mathcal{O}_{A, S}^{\times} / p\right)^{\chi} \rightarrow\left(I_{A, S}^{p} / p\right)^{\chi} \rightarrow\left(\mathrm{Cl}\left(\mathcal{O}_{A}[1 / p]\right) / p\right)^{\chi} \rightarrow 0 .
$$

Let $u=u(A, \chi):=\operatorname{dim}_{\mathbb{F}_{p}}\left(\mathcal{O}_{A}[1 / p]^{\times} / p\right)^{\chi}$.

Lemma 7.1. Assume that $\mu_{p}(A)^{\chi}=0$.

(a) Let $S_{\infty}$ (resp. $\left.S_{p}\right)$ be the set of places of A lying above $\infty$ (resp. $\left.p\right)$. Then

$$
u=\operatorname{dim}_{\mathbb{F}_{p}}\left(\mathbb{F}_{p}^{S_{\infty}}\right)^{\chi}+\operatorname{dim}_{\mathbb{F}_{p}}\left(\mathbb{F}_{p}^{S_{p}}\right)^{\chi}- \begin{cases}1, & \text { if } \chi=1 ; \\ 0, & \text { otherwise. }\end{cases}
$$

(b) We have

$$
\operatorname{dim}_{\mathbb{F}_{p}}\left(\mathcal{O}_{A, S}^{\times} / p\right)^{\chi}=\operatorname{dim}_{\mathbb{F}_{p}}\left(I_{A, S}^{p} / p\right)^{\chi}+u .
$$

(c) The quantity $\operatorname{dim}_{\mathbb{F}_{p}}\left(I_{A, S}^{p} / p\right)^{\chi}$ can be made arbitrarily large by choosing $S$ appropriately.

Proof.

(a) The Dirichlet $S$-unit theorem implies that the abelian group $\mathcal{O}_{A}[1 / p]^{\times}$is finitely generated with torsion subgroup $\mu(A)$. Let $M$ be the $\mathbb{Z} G$-module $\mathcal{O}_{A}[1 / p]^{\times} / \mu(A)$. Tensoring the exact sequence

$$
\mu(A) \longrightarrow \mathcal{O}_{A}[1 / p]^{\times} \longrightarrow M \longrightarrow 0
$$

with $\mathbb{F}_{p}$ and taking $\chi$-isotypic components yields

$$
0 \longrightarrow\left(\mathcal{O}_{A}[1 / p]^{\times} / p\right)^{\chi} \longrightarrow(M / p)^{\chi} \longrightarrow 0
$$

so $u=\operatorname{dim}_{\mathbb{F}_{p}}(M / p)^{\chi}$. 
On the other hand, the proof of the Dirichlet $S$-unit theorem yields

$$
M \otimes \mathbb{R} \simeq \mathcal{O}_{A}[1 / p]^{\times} \otimes \mathbb{R} \simeq\left(\mathbb{R}^{S_{\infty} \cup S_{p}}\right)_{\text {sum } 0}
$$

as $\mathbb{R} G$-modules. A $\mathbb{Z}_{(p)} G$-module that is free of finite rank over $\mathbb{Z}_{(p)}$ is determined by its character, so

$$
M \otimes \mathbb{Z}_{(p)} \simeq\left(\mathbb{Z}_{(p)}^{S_{\infty} \cup S_{p}}\right)_{\text {sum } 0}
$$

as $\mathbb{Z}_{(p)} G$-modules. Both sides are free over $\mathbb{Z}_{(p)}$, so we may tensor with $\mathbb{F}_{p}$ to obtain

$$
M / p \simeq\left(\mathbb{F}_{p}^{S_{\infty} \cup S_{p}}\right)_{\text {sum } 0}
$$

as $\mathbb{F}_{p} G$-modules. In other words, there is an exact sequence

$$
0 \longrightarrow M / p \longrightarrow \mathbb{F}_{p}^{S_{\infty}} \oplus \mathbb{F}_{p}^{S_{p}} \longrightarrow \mathbb{F}_{p} \longrightarrow 0
$$

Taking dimensions of the $\chi$-components yields the formula for $u$.

(b) The composition $G \stackrel{\chi}{\rightarrow}(\mathbb{Z} / p \mathbb{Z})^{\times} \hookrightarrow \mathbb{Z}_{p}^{\times}$lets us identify $\chi$ with a $\mathbb{Z}_{p}$-representation of $G$. Tensor (3) with $\mathbb{Z}_{p}$, and take $\chi$-isotypic components:

$0 \rightarrow\left(\mathcal{O}_{A}[1 / p]^{\times} \otimes \mathbb{Z}_{p}\right)^{\chi} \rightarrow\left(\mathcal{O}_{A, S}^{\times} \otimes \mathbb{Z}_{p}\right)^{\chi} \rightarrow\left(I_{A, S}^{p} \otimes \mathbb{Z}_{p}\right)^{\chi} \rightarrow\left(\mathrm{Cl}\left(\mathcal{O}_{A}[1 / p]\right) \otimes \mathbb{Z}_{p}\right)^{\chi} \rightarrow 0$.

Since $\mu_{p}(A)^{\chi}=0$, the first three $\mathbb{Z}_{p}$-modules are free; on the other hand, the last is finite as a set. Take $\mathbb{Z}_{p}$-ranks. If $V$ is a $\mathbb{Z}_{p} G$-module such that $V^{\chi}$ is a free $\mathbb{Z}_{p}$-module of finite rank, then $\operatorname{dim}_{\mathbb{F}_{p}}(V / p)^{\chi}=\operatorname{rank}_{\mathbb{Z}_{p}} V^{\chi}$. This proves the formula.

(c) If $S$ contains $m$ rational primes that split completely in $A$, then $I_{A, S}^{p}$ contains $(\mathbb{Z} G)^{m}$, so $\operatorname{dim}_{\mathbb{F}_{p}}\left(I_{A, S}^{p} / p\right)^{\chi} \geq m$, and $m$ can be chosen arbitrarily large.

Sequence (44) and Lemma 7.1(b,c) imply that $\left(\mathrm{Cl}\left(\mathcal{O}_{A}[1 / p]\right) / p\right)^{\chi}$ is naturally the cokernel of a linear map $\mathbb{F}_{p}^{m+u} \rightarrow \mathbb{F}_{p}^{m}$ for arbitrarily large $m$. In Section 8 , we will vary $(A, \chi)$ in a family with constant $u$-value and conjecture that the distribution of $\left(\mathrm{Cl}\left(\mathcal{O}_{A}[1 / p]\right) / p\right)^{\chi}$ equals the limit as $m \rightarrow \infty$ of the distribution of the cokernel of a random linear map $\mathbb{F}_{p}^{m+u} \rightarrow \mathbb{F}_{p}^{m}$; the precise statement is Conjecture 8.2. For now, we mention that this limiting distribution and the limiting expected size of the cokernel are known:

Proposition 7.2. Fix a prime $p$ and an integer $u \geq 0$. For $m \geq 0$, let $N$ be a linear map $\mathbb{F}_{p}^{m+u} \rightarrow \mathbb{F}_{p}^{m}$ chosen uniformly at random, and let $\mathcal{N}_{p, u, m}$ be the random variable $\operatorname{dim}_{\mathbb{F}_{p}} \operatorname{coker}(N)$. Then

(a) For each $r \geq 0$,

$$
\lim _{m \rightarrow \infty} \operatorname{Prob}\left(\mathcal{N}_{p, u, m}=r\right)=\alpha_{p, u, r}:=\frac{\prod_{i=r+1}^{\infty}\left(1-p^{-i}\right)}{p^{r(u+r)} \prod_{i=1}^{r+u}\left(1-p^{-i}\right)} .
$$

(b) We have $\sum_{r=0}^{\infty} \alpha_{p, u, r}=1$.

(c) We have $\sum_{r=0}^{\infty} p^{r} \alpha_{p, u, r}=1+p^{-u}$.

Proof.

(a) This is KL75, Theorem 1].

(b) This is the $q=1 / p$ and $\alpha=0$ case of [CL84, Corollary 6.7].

(c) This is the $q=1 / p$ and $\alpha=1$ case of [CL84, Corollary 6.7].

Remark 7.3. The constant $\alpha_{p, u, r}$ appeared also in [CL84, Theorem 6.3], as the $u$-probability that a random finite abelian $p$-group has $p$-rank $r$. The connection between $u$-probabilities and coranks of random matrices was made in [FW89. 


\section{Heuristics For Class groups And Even $K$-Groups ASSOCIATEd to QuAdratiC FIELDS}

8.1. Calculation of $u$. We now specialize Section 7 to the setting of Section 3 , Thus $F$ is $\mathbb{Q}(\sqrt{d})$ for some $d \in \mathbb{Q}^{\times}$such that $d$ and $p^{*}$ are independent in $\mathbb{Q}^{\times} / \mathbb{Q}^{\times 2}$; by multiplying $d$ by a square, we may assume that $d$ is a fundamental discriminant: $d=d_{F}$. Also, $E:=F\left(\zeta_{p}\right)$ and $\chi:=\chi_{-n,-1}$. Define $u(E, \chi)$ as in the sentence before Lemma 7.1 .

Proposition 8.1. The value $u(E, \chi)$ is given by the following table:

\begin{tabular}{c|c||c|c} 
& & $d>0$ & $d<0$ \\
\hline \hline$n$ even & $n \equiv 0(\bmod p-1), d \in \mathbb{Q}_{p}^{\times 2}$ & 2 & 1 \\
& $n \equiv \frac{p-1}{2}(\bmod p-1), p^{*} d \in \mathbb{Q}_{p}^{\times 2}$ & 2 & 1 \\
& all other cases & 1 & 0 \\
\hline$n$ odd & $n \equiv \frac{p-1}{2}(\bmod p-1), p^{*} d \in \mathbb{Q}_{p}^{\times 2}$ & 1 & 2 \\
& all other cases & 0 & 1
\end{tabular}

Proof. Because $\operatorname{Gal}\left(E / \mathbb{Q}\left(\zeta_{p}\right)\right)$ acts on $\mu_{p}(E)$ as +1 , we have $\mu_{p}(E)^{\chi}=0$, so Lemma 7.1(国) applies with $\chi=\chi_{-n,-1}$. The complex conjugation in $\operatorname{Gal}(E / \mathbb{Q}) \simeq(\mathbb{Z} / p \mathbb{Z})^{\times} \times\{ \pm 1\}$ is $c:=(-1, \pm 1)$, where the \pm 1 is +1 if $F$ is real, and -1 if $F$ is imaginary. The $G$-set $S_{\infty}$ is isomorphic to $G /\langle c\rangle$, so $\mathbb{F}_{p}^{S_{\infty}}$ is the $c$-invariant subrepresentation of the regular representation $\mathbb{F}_{p} G$. The multiplicity of $\chi$ in $\mathbb{F}_{p} G$ is 1 , so the multiplicity of $\chi$ in $\mathbb{F}_{p}^{S_{\infty}}$ is 1 or 0 , according to whether $\chi(c)$ is 1 or -1 . By definition of $\chi_{-n,-1}$, we have $\chi(c)=(-1)^{-n} \operatorname{sgn}(d)$, so

$$
\operatorname{dim}_{\mathbb{F}_{p}}\left(\mathbb{F}_{p}^{S_{\infty}}\right)^{\chi}= \begin{cases}1, & \text { if }(-1)^{n} d>0 ; \\ 0, & \text { if }(-1)^{n} d<0 .\end{cases}
$$

Next, Corollary 4.2 implies

$$
\operatorname{dim}_{\mathbb{F}_{p}}\left(\mathbb{F}_{p}^{S_{p}}\right)^{\chi}=\operatorname{dim}_{\mathbb{F}_{p}}\left(\operatorname{Br}\left(\mathcal{O}_{E}[1 / p]\right)_{p}\right)^{\chi},
$$

which is given by Proposition 4.4. The third term in Lemma 7.1(可) is 0 since $\chi \neq 1$.

8.2. Distribution. Suppose that $\mathscr{F}$ is a family of quadratic fields. For $X>0$, let $\mathscr{F}<X$ be the set of $F \in \mathscr{F}$ such that $\left|d_{F}\right|<X$. For any function $\gamma: \mathscr{F} \rightarrow \mathbb{Z}_{\geq 0}$, define the following notation:

$$
\begin{aligned}
\operatorname{Prob}(\gamma(F)=r) & :=\lim _{X \rightarrow \infty} \frac{\#\left\{F \in \mathscr{F}_{<X}: \gamma(F)=r\right\}}{\# \mathscr{F}_{<X}}, \\
\text { Average }(\gamma) & :=\lim _{X \rightarrow \infty} \frac{\sum_{F \in \mathscr{F}_{<X}} \gamma(F)}{\# \mathscr{F}_{<X}} .
\end{aligned}
$$

Our heuristic is formalized in the following statement.

Conjecture 8.2 (Distribution of class group components). Fix one of the ten boxes (below and right of the double lines) in the table of Proposition 8.1, and fix a corresponding $n \geq 1$. Let $F$ vary over the family $\mathscr{F}$ of quadratic fields with d satisfying the conditions defining that box, and let $u$ be as calculated in Proposition 8.1. Then the distribution of $\left(\mathrm{Cl}\left(\mathcal{O}_{E}[1 / p]\right) / p\right)^{\chi}$ equals the limit as $m \rightarrow \infty$ of the distribution of a cokernel of a random linear map $\mathbb{F}_{p}^{m+u} \rightarrow$ $\mathbb{F}_{p}^{m}$; by this we mean, in the notation of Proposition 7.2. that for each $r \in \mathbb{Z}_{\geq 0}$,

$$
\operatorname{Prob}\left(\operatorname{dim}_{\mathbb{F}_{p}}\left(\mathrm{Cl}\left(\mathcal{O}_{E}[1 / p]\right) / p\right)^{\chi}=r\right)=\lim _{m \rightarrow \infty} \operatorname{Prob}\left(\mathcal{N}_{p, u, m}=r\right),
$$


which by Proposition 7.2(国) equals $\alpha_{p, u, r}$.

If Conjecture 8.2 holds, then substituting it and Proposition 4.4 (with Remark 4.5) into Theorem 3.1 yields the following:

Conjecture 8.3 (Distribution of $K$-groups modulo $p$ in residue classes). Fix $n \geq 1$ and an odd prime $p$ and $r \geq 0$. As $F$ ranges over the quadratic fields $\mathbb{Q}(\sqrt{d})$ with $d$ satisfying the conditions defining a box below, Prob $\left(\operatorname{dim}_{\mathbb{F}_{p}}\left(K_{2 n}\left(\mathcal{O}_{F}\right) / p\right)^{-}=r\right)$ is as follows:

\begin{tabular}{c|c||c|c} 
& & $d>0$ & $d<0$ \\
\hline \hline$n$ even & $n \equiv 0(\bmod p-1), d \in \mathbb{Q}_{p}^{\times 2}$ & $\alpha_{p, 2, r-1}$ & $\alpha_{p, 1, r-1}$ \\
& $n \equiv \frac{p-1}{2}(\bmod p-1), p^{*} d \in \mathbb{Q}_{p}^{\times 2}$ & $\alpha_{p, 2, r-1}$ & $\alpha_{p, 1, r-1}$ \\
& all other cases & $\alpha_{p, 1, r}$ & $\alpha_{p, 0, r}$ \\
\hline$n$ odd & $n \equiv \frac{p-1}{2}(\bmod p-1), p^{*} d \in \mathbb{Q}_{p}^{\times 2}$ & $\alpha_{p, 1, r-1}$ & $\alpha_{p, 2, r-1}$ \\
& all other cases & $\alpha_{p, 0, r}$ & $\alpha_{p, 1, r}$
\end{tabular}

The distribution of $\operatorname{dim}_{\mathbb{F}_{p}} K_{2 n}\left(\mathcal{O}_{F}\right) / p$ is the distribution of $\operatorname{dim}_{\mathbb{F}_{p}}\left(K_{2 n}\left(\mathcal{O}_{F}\right) / p\right)^{-}$shifted by the constant $\kappa_{2 n, p}=\operatorname{dim}_{\mathbb{F}_{p}} K_{2 n}(\mathbb{Z})_{p}$ of Section 5 .

In Conjecture 8.3, the family of fields was defined by specifying both the sign of $d$ and a $p$-adic condition on $d$. To get the analogous probabilities for a larger family in which only the sign of $d$ is specified, we can take a weighted combination of probabilities from Conjecture 8.3.

Example 8.4. Suppose that $n \equiv 0(\bmod p-1)$ and $\mathscr{F}$ is the family of real quadratic fields $F$. By the first and third entries of the $d>0$ column of the table of Conjecture 8.3 , $\operatorname{Prob}\left(\operatorname{dim}_{\mathbb{F}_{p}}\left(K_{2 n}\left(\mathcal{O}_{F}\right) / p\right)^{-}=r\right)$ equals

$$
\operatorname{Prob}\left(d \in \mathbb{Q}_{p}^{\times 2}\right) \alpha_{p, 2, r-1}+\operatorname{Prob}\left(d \notin \mathbb{Q}_{p}^{\times 2}\right) \alpha_{p, 1, r} .
$$

Since $p^{2} \nmid d$, we have $d \in \mathbb{Q}_{p}^{\times 2}$ if and only if $(d \bmod p) \in \mathbb{F}_{p}^{\times 2}$; this condition is satisfied by $d$ lying in $p \cdot(p-1) / 2$ of the $p^{2}-1$ nonzero residue classes modulo $p^{2}$. The discriminant $d$ is equally likely to be in any of the $p^{2}-1$ nonzero residue classes modulo $p^{2}$, as follows, for example, from [Pra58, (1)], so

$$
\operatorname{Prob}\left(d \in \mathbb{Q}_{p}^{\times 2}\right)=\frac{p(p-1) / 2}{p^{2}-1}=\frac{p}{2 p+2} .
$$

Substituting this and the complementary probability into (5) yields

$$
\operatorname{Prob}\left(\operatorname{dim}_{\mathbb{F}_{p}}\left(K_{2 n}\left(\mathcal{O}_{F}\right) / p\right)^{-}=r\right)=\frac{p}{2 p+2} \alpha_{p, 2, r-1}+\frac{p+2}{2 p+2} \alpha_{p, 1, r} .
$$

Similar calculations show that Conjecture 8.3 implies all ten cases in Conjecture 8.5 below.

Conjecture 8.5 (Distribution of $K$-groups modulo $p$; cf. Conjecture 1.1). Fix $n \geq 1$ and an odd prime $p$ and $r \geq 0$. As $F$ ranges over all real (resp. imaginary) quadratic fields, $\operatorname{Prob}\left(\operatorname{dim}_{\mathbb{F}_{p}}\left(K_{2 n}\left(\mathcal{O}_{F}\right) / p\right)^{-}=r\right)$ is given in the following table by the entry in the row determined by $n$ and column determined by the signature: 


\begin{tabular}{c|c||c|c} 
& & real & imaginary \\
\hline \hline$n$ even & $n \equiv 0(\bmod p-1)$ & $\frac{p}{2 p+2} \alpha_{p, 2, r-1}+\frac{p+2}{2 p+2} \alpha_{p, 1, r}$ & $\frac{p}{2 p+2} \alpha_{p, 1, r-1}+\frac{p+2}{2 p+2} \alpha_{p, 0, r}$ \\
& $n \equiv \frac{p-1}{2}(\bmod p-1)$ & $\frac{1}{2 p+2} \alpha_{p, 2, r-1}+\frac{2 p+1}{2 p+2} \alpha_{p, 1, r}$ & $\frac{1}{2 p+2} \alpha_{p, 1, r-1}+\frac{2 p+1}{2 p+2} \alpha_{p, 0, r}$ \\
& all other cases & $\alpha_{p, 1, r}$ & $\alpha_{p, 0, r}$ \\
\hline$n$ odd & $n \equiv \frac{p-1}{2}(\bmod p-1)$ & $\frac{1}{2 p+2} \alpha_{p, 1, r-1}+\frac{2 p+1}{2 p+2} \alpha_{p, 0, r}$ & $\frac{1}{2 p+2} \alpha_{p, 2, r-1}+\frac{2 p+1}{2 p+2} \alpha_{p, 1, r}$ \\
& all other cases & $\alpha_{p, 0, r}$ & $\alpha_{p, 1, r}$.
\end{tabular}

The distribution of $\operatorname{dim}_{\mathbb{F}_{p}} K_{2 n}\left(\mathcal{O}_{F}\right) / p$ is the distribution of $\operatorname{dim}_{\mathbb{F}_{p}}\left(K_{2 n}\left(\mathcal{O}_{F}\right) / p\right)^{-}$shifted by $\kappa_{2 n, p}$.

8.3. Average order. Proposition 8.1 combined with the reasoning of Section 7 (Proposition 7.2 (C) , in particular) suggests the following statement:

Conjecture 8.6 (Average order of class group components in residue classes). Fix an odd prime $p$. The average order of $\left(\mathrm{Cl}\left(\mathcal{O}_{E}[1 / p]\right) / p\right)^{\chi}$ for $F$ ranging over the quadratic fields $\mathbb{Q}(\sqrt{d})$ satisfying the conditions defining a box below is as follows:

\begin{tabular}{c|c||c|c} 
& & $d>0$ & $d<0$ \\
\hline \hline$n$ even & $n \equiv 0(\bmod p-1), d \in \mathbb{Q}_{p}^{\times 2}$ & $1+p^{-2}$ & $1+p^{-1}$ \\
& $n \equiv \frac{p-1}{2}(\bmod p-1), p^{*} d \in \mathbb{Q}_{p}^{\times 2}$ & $1+p^{-2}$ & $1+p^{-1}$ \\
& all other cases & $1+p^{-1}$ & 2 \\
\hline$n$ odd & $n \equiv \frac{p-1}{2}(\bmod p-1), p^{*} d \in \mathbb{Q}_{p}^{\times 2}$ & $1+p^{-1}$ & $1+p^{-2}$ \\
& all other cases & 2 & $1+p^{-1}$
\end{tabular}

Conjecture 8.6, in turn, would imply the following:

Conjecture 8.7 (Average order of $K$-groups modulo $p$ in residue classes). Fix $n \geq 1$ and an odd prime $p$. The average order of $\left(K_{2 n}\left(\mathcal{O}_{F}\right) / p\right)^{-}$for $F$ ranging over the quadratic fields $\mathbb{Q}(\sqrt{d})$ satisfying the conditions defining a box below is as follows:

\begin{tabular}{c|c||c|c} 
& & $d>0$ & $d<0$ \\
\hline \hline$n$ even & $n \equiv 0(\bmod p-1), d \in \mathbb{Q}_{p}^{\times 2}$ & $p+p^{-1}$ & $p+1$ \\
& $n \equiv \frac{p-1}{2}(\bmod p-1), p^{*} d \in \mathbb{Q}_{p}^{\times 2}$ & $p+p^{-1}$ & $p+1$ \\
& all other cases & $1+p^{-1}$ & 2 \\
\hline$n$ odd & $n \equiv \frac{p-1}{2}(\bmod p-1), p^{*} d \in \mathbb{Q}_{p}^{\times 2}$ & $p+1$ & $p+p^{-1}$ \\
& all other cases & 2 & $1+p^{-1}$
\end{tabular}

To get the average order of $K_{2 n}\left(\mathcal{O}_{F}\right) / p$ itself, multiply each entry by $p^{\kappa_{2 n, p}}$.

Conjecture 8.7 implies Conjecture 8.8 below for the family of all quadratic fields of given signature, by taking weighted combinations of averages. For example, for $n \equiv 0(\bmod p-1)$ and real quadratic fields (cf. Example 8.4),

$$
\begin{aligned}
\text { Average } \#\left(K_{2 n}\left(\mathcal{O}_{F}\right) / p\right)^{-} & =\operatorname{Prob}\left(d \in \mathbb{Q}_{p}^{\times 2}\right)\left(p+p^{-1}\right)+\operatorname{Prob}\left(d \notin \mathbb{Q}_{p}^{\times 2}\right)\left(1+p^{-1}\right) \\
& =\frac{p}{2 p+2}\left(p+p^{-1}\right)+\frac{p+2}{2 p+2}\left(1+p^{-1}\right) \\
& =\frac{p^{3}+p^{2}+4 p+2}{2 p^{2}+2 p}
\end{aligned}
$$


Conjecture 8.8 (Average order of $K$-groups modulo $p$ ). Fix $n \geq 1$ and an odd prime $p$. The average order of $\left(K_{2 n}\left(\mathcal{O}_{F}\right) / p\right)^{-}$for $F$ ranging over all real (resp. imaginary) quadratic fields is given in the following table by the entry in the row determined by $n$ and column determined by the signature:

\begin{tabular}{c|c||c|c} 
& & real & imaginary \\
\hline \hline$n$ even & $n \equiv 0(\bmod p-1)$ & $\frac{p^{3}+p^{2}+4 p+2}{2 p^{2}+2 p}$ & $\frac{p^{2}+3 p+4}{2 p+2}$ \\
& $n \equiv \frac{p-1}{2}(\bmod p-1)$ & $\frac{3 p^{2}+3 p+2}{2 p^{2}+2 p}$ & $\frac{5 p+3}{2 p+2}$ \\
& all other cases & $\frac{p+1}{p}$ & 2 \\
\hline \multirow{2}{*}{$n$ odd } & $n \equiv \frac{p-1}{2}(\bmod p-1)$ & $\frac{5 p+3}{2 p+2}$ & $\frac{3 p^{2}+3 p+2}{2 p^{2}+2 p}$ \\
& all other cases & 2 & $\frac{p+1}{p}$
\end{tabular}

To get the distribution of the order of $K_{2 n}\left(\mathcal{O}_{F}\right) / p$ itself, multiply each entry by $p^{\kappa_{2 n, p}}$.

Remark 8.9. It is not quite clear that Conjectures 8.2, 8.3, and 8.5, imply Conjectures 8.6, 8.7, and 8.8, respectively. For such implications, one would need to know that the contribution to each average from the rare cases of very large class groups or $K$-groups is negligible.

\section{The AVERAGE ORDER OF EVEN $K$-Groups MOdulO 3}

In this section, we prove Conjecture 8.6 for $p=3$; then Conjectures 8.7 and 8.8 for $p=3$ follow too; the latter becomes Theorem 1.2. For $p=3$, the table in Conjecture 8.6 to be verified simplifies to

\begin{tabular}{c|c||c|c} 
& & $d>0$ & $d<0$ \\
\hline \hline$n$ even & $d \in \mathbb{Q}_{3}^{\times 2}$ & $10 / 9$ & $4 / 3$ \\
& $d \notin \mathbb{Q}_{3}^{\times 2}$ & $4 / 3$ & 2 \\
\hline$n$ odd & $-3 d \in \mathbb{Q}_{3}^{\times 2}$ & $4 / 3$ & $10 / 9$ \\
& $-3 d \notin \mathbb{Q}_{3}^{\times 2}$ & 2 & $4 / 3$.
\end{tabular}

We have $E=F\left(\zeta_{3}\right)$ and $G=\operatorname{Gal}(E / \mathbb{Q}) \simeq(\mathbb{Z} / 3 \mathbb{Z})^{\times} \times\{ \pm 1\}$. Let $H:=$ ker $\chi$, which is generated by $\left(-1,(-1)^{n}\right)$. Let $K:=E^{H}$, which is $F$ if $n$ is even, and $F^{\prime}=\mathbb{Q}(\sqrt{-3 d})$ if $n$ is odd. For any $\mathbb{F}_{3}$-representation $V$ of $G$, we have $V^{H}=V^{G} \oplus V^{\chi}$. Apply this to $V=\operatorname{Cl}\left(\mathcal{O}_{E}[1 / 3]\right) / 3$, and use Lemma 2.3 twice to obtain

$$
\mathrm{Cl}\left(\mathcal{O}_{K}[1 / 3]\right) / 3 \simeq \mathrm{Cl}(\mathbb{Z}[1 / 3]) / 3 \oplus\left(\mathrm{Cl}\left(\mathcal{O}_{E}[1 / 3]\right) / 3\right)^{\chi} \simeq\left(\mathrm{Cl}\left(\mathcal{O}_{E}[1 / 3]\right) / 3\right)^{\chi},
$$

since $\mathrm{Cl}(\mathbb{Z}[1 / 3])$ is a quotient of $\mathrm{Cl}(\mathbb{Z})=0$. It remains to show that for each box, the average order of $\mathrm{Cl}\left(\mathcal{O}_{K}[1 / 3]\right) / 3$ is as given in the table above.

For fixed $n$, as $d$ ranges over fundamental discriminants of fixed sign in a fixed coset of $\mathbb{Q}_{3}^{\times 2}$ up to some bound, the field $K$ ranges over quadratic fields with fundamental discriminant of fixed sign in a fixed coset of $\mathbb{Q}_{3}^{\times 2}$ up to some bound (the same sign and coset if $n$ is even, or sign and coset multiplied by -3 if $n$ is odd). Thus it suffices to prove that the average order of $\mathrm{Cl}\left(\mathcal{O}_{K}[1 / 3]\right) / 3$ for such $K$ is given by the table

\begin{tabular}{c||cc} 
& $d_{K}>0$ & $d_{K}<0$ \\
\hline \hline$d_{K} \in \mathbb{Q}_{3}^{\times 2}$ & $10 / 9$ & $4 / 3$ \\
$d_{K} \notin \mathbb{Q}_{3}^{\times 2}$ & $4 / 3$ & 2. \\
& 12 &
\end{tabular}


Remark 9.1. There are four cosets of $\mathbb{Q}_{3}^{\times 2}$ in $\mathbb{Q}_{3}^{\times}$. Which coset contains a given fundamental discriminant $d_{K}$ is determined by whether $d_{K}$ is $1 \bmod 3,2 \bmod 3,3 \bmod 9$, or $6 \bmod 9$. In particular, $d_{K} \in \mathbb{Q}_{3}^{\times 2}$ if and only if $d_{K} \equiv 1(\bmod 3)$.

Remark 9.2. The results in Sections 9.1 to 9.3 have been generalized by the second author to compute the average size of $\mathrm{Cl}\left(\mathcal{O}_{K}[1 / S]\right) / 3$ for an arbitrary finite set of primes $S$ using essentially identical methods Kla17a]. Similar methods have been used to compute the average size of $\mathrm{Cl}\left(O_{K}[1 / p]\right) / 3$ when $p$ splits in $K$ [Woo18].

9.1. From class groups to cubic fields. To compute the average order of $\mathrm{Cl}\left(\mathcal{O}_{K}[1 / 3]\right) / 3$, we adapt the strategy of Davenport and Heilbronn [DH71], which relies on results involving the following setup.

Let $L$ be a degree 3 extension of $\mathbb{Q}$ whose Galois closure $M$ has Galois group $S_{3}$. Let $K$ be the quadratic resolvent of $L$, that is, the degree 2 subfield $M^{A_{3}}$.

Lemma 9.3. The following are equivalent:

(i) $M / K$ is unramified.

(ii) $d_{L}=d_{K}$.

(iii) $L / \mathbb{Q}$ is nowhere totally ramified.

Proof. Let $\mathfrak{f}$ be the conductor of the abelian extension $M / K$. By [Has30, Satz 3], $d_{L}=$ $N_{K / \mathbb{Q}}(\mathfrak{f}) d_{K}$; this proves (i) $\leftrightarrow(\mathrm{ii})$. Also, $d_{L}$ is an integer square times $d_{K}$ (see [Has30, (1)]); this proves (ii) $\leftrightarrow$ (iii). Finally, Coh00, Proposition 8.4.1] yields (ii) $\leftrightarrow$ (iv).

Throughout this section, we consider two cubic fields to be the same if they are abstractly isomorphic (so conjugate cubic fields are counted only as one).

Theorem 9.4 (cf. [Has30, Satz 7]). Fix a quadratic field $K$. Then the following are naturally in bijection:

(i) The set of index 3 subgroups of $\mathrm{Cl}\left(\mathcal{O}_{K}\right)$.

(ii) The set of unramified $\mathbb{Z} / 3 \mathbb{Z}$-extensions $M$ of $K$.

(iii) The set of cubic fields $L$ with $d_{L}=d_{K}$.

Proof. Class field theory gives (i) $\leftrightarrow$ (ii). The nontrivial element of $\operatorname{Gal}(K / \mathbb{Q})$ acts as -1 on $\mathrm{Cl}\left(\mathcal{O}_{K}\right)$, so each $M$ in (ii) is an $S_{3}$-extension of $\mathbb{Q}$. The map (ii) $\rightarrow$ (iii) sends $M$ to one of its cubic subfields $L$. The map (iii) $\rightarrow$ (ii) sends $L$ to its Galois closure $M$. That these are bijections follows from Lemma 9.3 (i) $\leftrightarrow$ (ii).

We are interested in $\mathrm{Cl}\left(\mathcal{O}_{K}[1 / 3]\right)$ instead of $\mathrm{Cl}\left(\mathcal{O}_{K}\right)$, so we need the following variant.

Corollary 9.5. Fix a quadratic field $K$. Then the following are naturally in bijection:

(i) The set of index 3 subgroups of $\mathrm{Cl}\left(\mathcal{O}_{K}\right)[1 / 3]$.

(ii) The set of unramified $\mathbb{Z} / 3 \mathbb{Z}$-extensions $M$ of $K$ such that the primes above 3 in $K$ split completely in $M / K$.

(iii) The set of cubic fields $L$ with $d_{L}=d_{K}$ such that if $d_{K} \in \mathbb{Q}_{3}^{\times 2}$ then 3 splits completely in $L / \mathbb{Q}$.

Proof. The group $\mathrm{Cl}\left(\mathcal{O}_{K}\right)[1 / 3]$ is the quotient of $\mathrm{Cl}\left(\mathcal{O}_{K}\right)$ by the group generated by the classes of the primes $\mathfrak{p} \mid 3$ in $K$. Thus we need to restrict the bijections in Theorem 9.4 to the index 3 subgroups $H$ containing these classes. Because the class field theory isomorphism 
$\mathrm{Cl}\left(\mathcal{O}_{K}\right) / H \simeq \operatorname{Gal}(M / K)$ sends $[\mathfrak{p}]$ to Frob Fhich is trivial if and only if $\mathfrak{p}$ splits in $M / K$, we obtain (i) $\leftrightarrow$ (ii). If 3 is inert or ramified in $K / \mathbb{Q}$, then the prime above 3 is of order dividing 2 in $\mathrm{Cl}\left(\mathcal{O}_{K}\right)$, so to require it to be in the index 3 subgroup is no condition. If 3 splits in $K / \mathbb{Q}$ (that is, $d_{K} \in \mathbb{Q}_{3}^{\times 2}$ ), then the primes above 3 in $K$ split in $M / K$ if and only if 3 splits completely in $M / \mathbb{Q}$, which is if and only if 3 splits completely in $L / \mathbb{Q}$.

Corollary 9.6. Let $K$ be a quadratic field.

(a) If $d_{K} \in \mathbb{Q}_{3}^{\times 2}$, then

$$
\# \mathrm{Cl}\left(\mathcal{O}_{K}[1 / 3]\right) / 3=2 \#\left\{\text { cubic fields } L \text { with } d_{L}=d_{K} \text { in which } 3 \text { splits }\right\}+1 .
$$

(b) If $d_{K} \notin \mathbb{Q}_{3}^{\times 2}$, then

$$
\# \mathrm{Cl}\left(\mathcal{O}_{K}[1 / 3]\right) / 3=2 \#\left\{\text { cubic fields } L \text { with } d_{L}=d_{K}\right\}+1 .
$$

Proof. For an elementary abelian 3-group $V$,

$$
\# V=2 \#\{\text { index } 3 \text { subgroups of } V\}+1 \text {. }
$$

Take $V=\operatorname{Cl}\left(\mathcal{O}_{K}[1 / 3]\right) / 3$, and apply Corollary $9.5(\mathrm{i}) \leftrightarrow(\mathrm{iii})$.

9.2. Counting quadratic fields. Fix a sign and a coset of $\mathbb{Q}_{3}^{\times 2}$ in $\mathbb{Q}_{3}^{\times}$; by Remark 9.1 , each of the four cosets is defined by a congruence condition mod 3 or mod 9 . Let $\mathscr{D}$ be the set of fundamental discriminants having this sign and lying in this coset. For $X>0$, define $\mathscr{D}_{<X}:=\{d \in \mathscr{D}:|d|<X\}$.

To compute the average of the appropriate left hand side in Corollary 9.6 as $K$ ranges over quadratic fields with $d_{K} \in \mathscr{D}$, we compute the average number of cubic fields appearing in the corresponding right hand side. That is, we need the limit as $X \rightarrow \infty$ of

$$
\frac{\sum_{K: d_{K} \in \mathscr{D}<X} \#\left\{\text { cubic fields } L \text { with } d_{L}=d_{K} \text { such that if } d_{K} \in \mathbb{Q}_{3}^{\times 2} \text { then } 3 \text { splits in } L\right\}}{\#\left\{\text { quadratic fields } K \text { such that } d_{K} \in \mathscr{D}_{<X}\right\}} .
$$

We first compute the denominator.

Proposition 9.7. The number of quadratic fields $K$ satisfying $\left|d_{K}\right|<X$ and prescribed sign and 3-adic congruence conditions is $\alpha_{2} X / \zeta(2)+o(X)$, where $\alpha_{2}$ is given by the following table:

\begin{tabular}{c||c|c} 
& $d_{K}>0$ & $d_{K}<0$ \\
\hline \hline$d_{K} \equiv 1(\bmod 3)$ & $3 / 16$ & $3 / 16$ \\
$d_{K} \equiv 2(\bmod 3)$ & $3 / 16$ & $3 / 16$ \\
$d_{K} \equiv 3(\bmod 9)$ & $1 / 16$ & $1 / 16$ \\
$d_{K} \equiv 6(\bmod 9)$ & $1 / 16$ & $1 / 16$.
\end{tabular}

Proof. Use an elementary squarefree sieve.

Remark 9.8. Even though many entries in the table of Proposition 9.7 coincide, it is stronger to give the asymptotics for the individual field families without merging them, and we need the stronger results. 
9.3. Counting cubic fields. By Lemma 9.3)(ii) $\leftrightarrow($ iii), the numerator in (6) equals the number of nowhere totally ramified cubic fields $L$ with $d_{L} \in \mathscr{D}_{<X}$ such that if $d_{L} \in \mathbb{Q}_{3}^{\times 2}$ then 3 splits completely in $L$. To compute this number, we follow the Davenport-Heilbronn approach, in the form of a refinement due to Bhargava, Shankar, and Tsimerman [BST13].

For every prime $p$, let $\widehat{\Sigma}_{p}$ be the set of maximal cubic $\mathbb{Z}_{p}$-orders that are not totally ramified, up to isomorphism. For $R \in \widehat{\Sigma}_{p}$, let $\operatorname{Disc}_{p}(R)$ be the power of $p$ generating the discriminant ideal of $R$.

Theorem 9.9. For each prime $p$ (including 2), let $\Sigma_{p} \subseteq \widehat{\Sigma}_{p}$. Suppose that $\Sigma_{p}=\widehat{\Sigma}_{p}$ for all $p$ outside a finite set $\mathcal{P}$. Define

$$
c_{p}:=\frac{p}{p+1} \sum_{R \in \Sigma_{p}} \frac{1}{\operatorname{Disc}_{p}(R)|\operatorname{Aut} R|} .
$$

(a) The number of nowhere totally ramified totally real cubic fields $L$ (up to isomorphism) such that $\left|d_{L}\right|<X$ and $\mathcal{O}_{L} \otimes \mathbb{Z}_{p} \in \Sigma_{p}$ for all $p \in \mathcal{P}$ is $\frac{1}{12 \zeta(2)}\left(\prod_{p \in \mathcal{P}} c_{p}\right) X+o(X)$.

(b) The number of nowhere totally ramified complex cubic fields $L$ (up to isomorphism) such that $\left|d_{L}\right|<X$ and $\mathcal{O}_{L} \otimes \mathbb{Z}_{p} \in \Sigma_{p}$ for all $p \in \mathcal{P}$ is $\frac{1}{4 \zeta(2)}\left(\prod_{p \in \mathcal{P}} c_{p}\right) X+o(X)$.

Proof. The definition of $c_{p}$ yields

$$
\frac{p-1}{p} \sum_{R \in \Sigma_{p}} \frac{1}{\operatorname{Disc}_{p}(R)|\operatorname{Aut} R|}=\left(1-\frac{1}{p^{2}}\right) c_{p} .
$$

For each $p \notin \mathcal{P}$, enumerating $\widehat{\Sigma}_{p}$ explicitly shows that $c_{p}=1$. Substituting this into BST13, Theorem 8] yields the result.

Corollary 9.10. The number of nowhere totally ramified cubic fields $L$ with $\left|d_{L}\right|<X$ satisfying prescribed sign and 3 -adic congruence conditions below such that if $d_{L} \equiv 1(\bmod 3)$ then 3 splits completely in $L$ is $\alpha_{3} X / \zeta(2)+o(X)$, where $\alpha_{3}$ is given by the following table:

\begin{tabular}{c||c|c} 
& $d_{L}>0$ & $d_{L}<0$ \\
\hline \hline$d_{L} \equiv 1(\bmod 3)$ & $1 / 96$ & $1 / 32$ \\
$d_{L} \equiv 2(\bmod 3)$ & $1 / 32$ & $3 / 32$ \\
$d_{L} \equiv 3(\bmod 9)$ & $1 / 96$ & $1 / 32$ \\
$d_{L} \equiv 6(\bmod 9)$ & $1 / 96$ & $1 / 32$.
\end{tabular}

Proof. We apply Theorem 9.9 with $\mathcal{P}=\{3\}$ and with $\Sigma_{3}$ tailored to the row. For the first row, let $\Sigma_{3}:=\left\{\mathbb{Z}_{3} \times \mathbb{Z}_{3} \times \mathbb{Z}_{3}\right\}$, so

$$
c_{3}=\frac{3}{4} \cdot \frac{1}{\operatorname{Disc}_{3}\left(\mathbb{Z}_{3} \times \mathbb{Z}_{3} \times \mathbb{Z}_{3}\right)\left|\operatorname{Aut}\left(\mathbb{Z}_{3} \times \mathbb{Z}_{3} \times \mathbb{Z}_{3}\right)\right|}=\frac{3}{4} \cdot \frac{1}{6}=\frac{1}{8} .
$$

For each other row, let $\Sigma_{3}:=\left\{\mathbb{Z}_{3} \times \mathbb{Z}_{3}\left(\sqrt{d_{L}}\right)\right\}$, and calculate $c_{3}$ similarly.

9.4. End of proof. Corollary 9.10 and Proposition 9.7 give the asymptotic behavior of the numerator and denominator, respectively, in (6) (see the first sentence of Section 9.3). Thus, as $X \rightarrow \infty$, the ratio (6) tends to $\alpha_{3} / \alpha_{2}$. Following Corollary 9.6, we multiply by 2 and add 1 to obtain the average order of $\mathrm{Cl}\left(\mathcal{O}_{K}[1 / 3]\right) / 3$ as $K$ varies over quadratic fields with $d_{K} \in \mathscr{D}$. For each signature, the answer is the same for each of the three nontrivial cosets of 
$\mathbb{Q}_{3}^{\times 2}$, so we combine them into a single entry in the table before Remark 9.1 . This completes the proof of Conjecture 8.6 for $p=3$, and hence also Conjectures 8.7 and 8.8 for $p=3$ and Theorem 1.2 .

\section{ACKNOWLEDGMENTS}

We thank the referee for taking the time to provide many helpful comments.

\section{REFERENCES}

[BKLPR15] Manjul Bhargava, Daniel M. Kane, Hendrik W. Lenstra Jr., Bjorn Poonen, and Eric Rains, Modeling the distribution of ranks, Selmer groups, and Shafarevich-Tate groups of elliptic curves, Camb. J. Math. 3 (2015), no. 3, 275-321. MR3393023

[BST13] Manjul Bhargava, Arul Shankar, and Jacob Tsimerman, On the Davenport-Heilbronn theorems and second order terms, Invent. Math. 193 (2013), no. 2, 439-499, DOI 10.1007/s00222-0120433-0. MR3090184

[BBH17] Nigel Boston, Michael R. Bush, and Farshid Hajir, Heuristics for p-class towers of imaginary quadratic fields, Math. Ann. 368 (2017), no. 1-2, 633-669, DOI 10.1007/s00208-016-1449-3. MR3651585

[BW17] Nigel Boston and Melanie Matchett Wood, Non-abelian Cohen-Lenstra heuristics over function fields, Compos. Math. 153 (2017), no. 7, 1372-1390, DOI 10.1112/S0010437X17007102. MR3705261

[Bro00] Jerzy Browkin, Tame kernels of quadratic number fields: numerical heuristics, Funct. Approx. Comment. Math. 28 (2000), 35-43. Dedicated to Włodzimierz Staś on the occasion of his 75th birthday. MR1823991

[BS82] J. Browkin and A. Schinzel, On Sylow 2-subgroups of $\mathrm{K}_{2} \mathrm{O}_{F}$ for quadratic number fields $F$, J. Reine Angew. Math. 331 (1982), 104-113, DOI 10.1515/crll.1982.331.104. MR647375

[BH11] J. P. Buhler and D. Harvey, Irregular primes to 163 million, Math. Comp. 80 (2011), no. 276, 2435-2444. MR2813369

[CGQ14] XiaoYun Cheng, XueJun Guo, and HouRong Qin, The densities for 3-ranks of tame kernels of cyclic cubic number fields, Sci. China Math. 57 (2014), no. 1, 43-47, DOI 10.1007/s11425-0134622-0. MR3146514

[Coh00] Henri Cohen, Advanced topics in computational number theory, Graduate Texts in Mathematics, vol. 193, Springer-Verlag, New York, 2000. MR1728313

[CL84] H. Cohen and H. W. Lenstra Jr., Heuristics on class groups of number fields, Number theory, Noordwijkerhout 1983 (Noordwijkerhout, 1983), Lecture Notes in Math., vol. 1068, Springer, Berlin, 1984, pp. 33-62, DOI 10.1007/BFb0099440. MR756082 (85j:11144)

[CM90] Henri Cohen and Jacques Martinet, Étude heuristique des groupes de classes des corps de nombres, J. Reine Angew. Math. 404 (1990), 39-76 (French). MR1037430 (91k:11097)

[DH71] H. Davenport and H. Heilbronn, On the density of discriminants of cubic fields. II, Proc. Roy. Soc. London Ser. A 322 (1971), no. 1551, 405-420. MR0491593 (58 \#10816)

[Del01] Christophe Delaunay, Heuristics on Tate-Shafarevitch groups of elliptic curves defined over $\mathbb{Q}$, Experiment. Math. 10 (2001), no. 2, 191-196. MR1837670 (2003a:11065)

[Del07] Christophe Delaunay, Heuristics on class groups and on Tate-Shafarevich groups: the magic of the Cohen-Lenstra heuristics, Ranks of elliptic curves and random matrix theory, London Math. Soc. Lecture Note Ser., vol. 341, Cambridge Univ. Press, Cambridge, 2007, pp. 323-340. MR2322355 (2008i:11089)

[DJ14] Christophe Delaunay and Frédéric Jouhet, $p^{\ell}$-torsion points in finite abelian groups and combinatorial identities, Adv. Math. 258 (2014), 13-45, DOI 10.1016/j.aim.2014.02.033. MR3190422

[FK07] Étienne Fouvry and Jürgen Klüners, On the 4-rank of class groups of quadratic number fields, Invent. Math. 167 (2007), no. 3, 455-513, DOI 10.1007/s00222-006-0021-2. MR2276261 (2007k:11187) 
[FW89] Eduardo Friedman and Lawrence C. Washington, On the distribution of divisor class groups of curves over a finite field, Théorie des nombres (Quebec, PQ, 1987), de Gruyter, Berlin, 1989, pp. 227-239. MR1024565 (91e:11138)

[Guo09] Xuejun Guo, On the 4-rank of tame kernels of quadratic number fields, Acta Arith. 136 (2009), no. 2, 135-149, DOI 10.4064/aa136-2-3. MR2475682

[Has30] Helmut Hasse, Arithmetische Theorie der kubischen Zahlkörper auf klassenkörpertheoretischer Grundlage, Math. Z. 31 (1930), no. 1, 565-582, DOI 10.1007/BF01246435 (German). MR1545136

[Kla17a] Zev Klagsbrun, Davenport-Heilbronn theorems for quotients of class groups, June 26, 2017. Preprint, arXiv:1701.02834v2 .

[Kla17b] Zev Klagsbrun, The average sizes of two-torsion subgroups in quotients of class groups of cubic fields, September 19, 2017. Preprint, arXiv:1701.02838v3.

[KL75] I. N. Kovalenko and A. A. Levitskaja, Limiting behavior of the number of solutions of a system of random linear equations over a finite field and a finite ring, Dokl. Akad. Nauk SSSR 221 (1975), no. 4, 778-781 (Russian). MR0380957

[Poo17] Bjorn Poonen, Rational points on varieties, Graduate Studies in Mathematics, vol. 186, American Mathematical Society, Providence, RI, 2017. MR3729254

[PR12] Bjorn Poonen and Eric Rains, Random maximal isotropic subspaces and Selmer groups, J. Amer. Math. Soc. 25 (2012), no. 1, 245-269, DOI 10.1090/S0894-0347-2011-00710-8. MR2833483

[Pra58] Karl Prachar, Über die kleinste quadratfreie Zahl einer arithmetischen Reihe, Monatsh. Math. 62 (1958), 173-176. MR0092806

[Tat76] John Tate, Relations between $K_{2}$ and Galois cohomology, Invent. Math. 36 (1976), 257-274. MR0429837 (55 \#2847)

[Wei05] Charles Weibel, Algebraic K-theory of rings of integers in local and global fields, Handbook of $K$ theory. Vol. 1, 2, Springer, Berlin, 2005, pp. 139-190, DOI 10.1007/3-540-27855-9_5. MR2181823

[Woo18] Melanie Matchett Wood, Cohen-Lenstra heuristics and local conditions, Res. Number Theory 4 (2018), no. 4, Art. 41, 22, DOI 10.1007/s40993-018-0134-x. MR3858473

Department of Mathematics, Baruch College, The City University of New York, One Bernard Baruch Way, New York, NY 10010-5526, USA

E-mail address: bruce.jordan@baruch.cuny.edu

Center for Communications Research, 4320 Westerra Court, San Diego, CA 92121-1969

E-mail address: zevklagsbrun@gmail.com

Department of Mathematics, Massachusetts Institute of Technology, Cambridge, Ma 02139-4307, USA

E-mail address: poonen@math.mit.edu

$U R L:$ http://math.mit.edu/ poonen/

Department of Mathematics, Princeton University, Fine Hall, Washington Road, PrinceTON, NJ 08544-1000, USA

E-mail address: cmcls@princeton.edu

Center for Communications Research, 805 Bunn Drive, Princeton, NJ 08540-1966, Usa

E-mail address: ykzaytm@idaccr.org 